\title{
Análisis de la gobernanza en la implementación del modelo comunitario de salud mental en Chile
}

\author{
Alberto Minoletti, ${ }^{1}$ Rafael Sepúlveda, ${ }^{1}$ Mauricio Gómez, ${ }^{2}$ Olga Toro, ${ }^{1}$ \\ Matías Irarrázabal, ${ }^{3}$ Rodrigo Díaz, ${ }^{4}$ Viviana Hernández ${ }^{2}$ y Susana Chacón ${ }^{4}$
}

Forma de citar Minoletti A, Sepúlveda R, Gómez M, Toro O, Irarrázabal M, Díaz R, et al. Análisis de la gobernanza en la implementación del modelo comunitario de salud mental en Chile. Rev Panam Salud Publica. 2018;42:e131. https://doi.org/10.26633/RPSP.2018.131

RESUMEN El presente artículo describe y analiza el rol que ha jugado la gobernanza en el desarrollo del modelo comunitario de salud mental en Chile. Se describen y analizan cinco dimensiones de la gobernanza: 1) el desarrollo de una serie de estrategias para favorecer la participación de los usuarios de servicios de salud mental ha logrado resultados insuficientes; 2) la implementación de tres planes nacionales de salud mental, que han mantenido la misma visión estratégica a lo largo de tres décadas, han contribuido a aumentar la accesibilidad a la atención comunitaria de las personas con enfermedades mentales y disminuir el rol de los hospitales psiquiátricos; 3) la inexistencia de una ley de salud mental persiste aún como un desafío, aunque haya sido parcialmente compensada por distintas leyes y decretos; 4) las regulaciones en la asignación de los recursos financieros, la formación del recurso humano y las directrices técnicas han contribuido a mejorar el acceso y la calidad de la atención; y 5) la construcción progresiva de un sistema de información en salud mental ha permitido conocer principalmente las tasas de acceso, las brechas de tratamiento y las inequidades geográficas. La experiencia chilena confirma la importancia de aplicar un conjunto de estrategias y mecanismos de gobernanza, para favorecer el desarrollo y el funcionamiento de los servicios de salud mental. El tipo de estrategias y mecanismos de gobernanza podrán variar en los distintos países según los contextos y las ventanas de oportunidades.

Palabras clave Gobernanza; servicios comunitarios de salud mental; política de salud; Chile.

La gobernanza implica proteger el interés público y requiere una acción tanto política como técnica que concilie demandas

\footnotetext{
Unidad de Salud Mental, Instituto de Salud Poblacional (Escuela de Salud Pública Dr. Salvador Allende), Facultad de Medicina, Universidad de Chile, Santiago, Chile. Enviar correspondencia a Alberto Minoletti, aminoletti2@gmail.com

2 Subsecretaría de Salud Pública, Ministerio de Salud, Gobierno de Chile, Santiago, Chile.
}

por recursos limitados en circunstancias siempre cambiantes y es, por lo tanto, uno de los componentes más críticos y

\footnotetext{
Departamento de Psiquiatría Norte, Hospital Clínico de la Universidad de Chile, Santiago, Chile.

4 Subsecretaría de Redes Asistenciales, Ministerio de Salud, Gobierno de Chile, Santiago, Chile
}

complejos de cualquier sistema de salud mental (1). Incluye funciones de supervisión y guía para todo el sistema de salud, la formulación de marcos estratégicos de políticas, la construcción de alianzas, la regulación y la rendición de cuentas (1).

El papel de la gobernanza en los sistemas de salud mental está reconocido en la Estrategia para la Cobertura Universal de Salud y en los Planes de Acción de la

Este es un artículo de acceso abierto distribuido bajo los términos de la licencia Creative Commons Attribution-NonCommercial-NoDerivs 3.0 IGO, que permite su uso, distribución y reproducción en cualquier medio, siempre que el trabajo original se cite de la manera adecuada. No se permiten modificaciones a los artículos ni su uso comercial. Al reproducir un artículo no debe haber ningún indicio de que la OPS o el artículo avalan a una organización o un producto específico. El uso del logo de la OPS no está permitido. Esta leyenda debe conservarse, junto con la URL original del artículo. 
Organización Panamericana de la Salud (OPS) y de la Organización Mundial de la Salud (OMS) (2-4). Esta impulsa su fortalecimiento en salud mental mediante la formulación e implementación de políticas, planes y leyes, y la promoción de servicios integrales, integrados y receptivos de salud mental y atención social basados en la comunidad $(2,4)$.

La implementación de servicios comunitarios que respondan en forma creciente a las necesidades de salud mental de la población es, a su vez, un facilitador para la gobernanza de los sistemas de atención en los países en vías de desarrollo (5). La integración de la salud mental en la atención primaria de salud (APS) facilita el acceso equitativo, la detección, el tratamiento, la rehabilitación y la recuperación de las personas con problemas de salud mental $(5,6)$.

Las leyes y políticas de salud mental que promueven la descentralización de la atención son un paso importante para la protección de las personas con trastornos mentales (5). En países de ingresos medios, el desarrollo de un modelo comunitario tiene el potencial de reducir las brechas de tratamiento y aumentar la cobertura y, de esta manera, mejorar las inequidades en el acceso a la atención, entre otros beneficios (7).

El presente artículo describe y analiza el rol que ha jugado la gobernanza en el desarrollo del modelo comunitario de salud mental en Chile.

\section{DESCRIPCIÓN DE LA GOBERNANZA DEL SISTEMA DE SALUD MENTAL EN CHILE}

Chile tiene un sistema público de salud que atiende al $74 \%$ de la población. Durante las últimas tres décadas, se han desarrollado servicios de salud mental integrados a este sistema, lo que logró disminuir el rol asistencial de los hospitales psiquiátricos y aumentar el número de personas atendidas en los dispositivos comunitarios y de APS (cuadros 1 y 2).

La gobernanza del sistema de salud en Chile posee diversas dimensiones y para su descripción se seleccionaron cinco de ellas que son de uso frecuente en la literatura comparada $(2,5,8-10)$.

\section{Mecanismos de participación}

La participación social ha sido incluida en los tres planes nacionales de salud mental, presentados en los años 1993, 2000 y 2017. El primer plan (11) destaca como objetivo "asegurar espacios que posibiliten la presencia de la comunidad y sus distintas formas de organización en la programación, ejecución y evaluación de las acciones de salud mental" y define mecanismos de participación a nivel de la gestión de las áreas de salud y en los centros de atención. El segundo plan (12) reitera planteamientos similares y prioriza "la participación activa de los usuarios, pacientes y familiares" en las distintas instancias de gestión de la red de servicios de salud mental y define las "responsabilidades de los equipos de salud para facilitar y estimular el protagonismo de los usuarios y familiares".

Desde el lanzamiento del primer plan nacional, se han implementado una serie de estrategias para favorecer la participación y asociatividad de los usuarios de servicios de salud mental y sus familias; sin embargo, el nivel de participación es bajo. De acuerdo a datos del 2013, el 1,6\% de los consultantes de salud mental participa en organizaciones de usuarios, $13,1 \%$ de estas organizaciones participa en la planificación de servicios de salud mental y $11,7 \%$ en las comisiones de protección de personas con enfermedades mentales (13).

\section{CUADRO 1. Comparación de camas/plazas para personas con trastornos mentales en distintos servicios con atención de 24 horas al día por 100000 beneficiarios del sistema público en Chile (1990 y 2014)}

\begin{tabular}{lrc}
\hline Camas/plazas para personas con trastornos mentales & 1990 & 2014 \\
\hline Estadía corta en hospitales generales (hasta 60 días) & 2,5 & 5,3 \\
Estadía corta en hospitales psiquiátricos (hasta 60 días) & 5,9 & 2,9 \\
Estadía mediana en hospitales psiquiátricos (hasta 1 año) & 0 & 0,9 \\
Estadía prolongada en hospitales psiquiátricos (indefinido) & 25,9 & 3,1 \\
Viviendas grupales comunitarias (indefinido) & 0 & 12,0 \\
\hline
\end{tabular}

Elaboración propia con base en datos del sistema de información del Ministerio de Salud de Chile, 2018.

CUADRO 2. Número de personas con trastornos mentales atendidas en distintos servicios por 100000 beneficiarios del sistema público en Chile (2004 y 2014)

\begin{tabular}{lrr}
\hline Personas con trastornos mentales atendidas & 2004 & 2014 \\
\hline Consultantes de atención primaria de salud & 2217 & 4559 \\
Consultantes de especialidad ambulatoria & 535 & 1014 \\
Personas ingresadas en hospitales generales & 55 & 86 \\
Personas ingresadas en hospitales psiquiátricos & 48 & 32 \\
\hline
\end{tabular}

Elaboración propia con base en datos del sistema de información del Ministerio de Salud de Chile, 2018.

\section{CUADRO 3. Principales estrategias para favorecer la participación y asociatividad de usuarios y familiares en Chile (1990-2017)}

\begin{tabular}{|c|c|}
\hline Nivel local (direcciones de las 29 áreas sanitarias del país) & Nivel nacional (Ministerio de Salud) \\
\hline $\begin{array}{l}\text { Orientaciones técnicas para clubes de integración social, para favorecer el } \\
\text { desarrollo de agrupaciones de personas con trastornos mentales graves }\end{array}$ & $\begin{array}{l}\text { Participación de representantes de diferentes agrupaciones de usuarios en reuniones } \\
\text { técnicas y jornadas de trabajo de la dirección de salud mental del Ministerio de Salud }\end{array}$ \\
\hline $\begin{array}{l}\text { Fondos de salud y otros sectores para apoyar iniciativas de grupos de usuarios } \\
\text { y familiares, con distribución mediante instancias locales }\end{array}$ & $\begin{array}{l}\text { Apoyo técnico y material para la conformación de una asociación nacional de grupos de } \\
\text { usuarios y familiares }\end{array}$ \\
\hline $\begin{array}{l}\text { Incentivos financieros para que los equipos de la APS promuevan la } \\
\text { conformación de grupos de ayuda mutua en salud mental }\end{array}$ & $\begin{array}{l}\text { Participación de usuarios y familiares en las comisiones de protección de personas con } \\
\text { enfermedades mentales }^{a}\end{array}$ \\
\hline Administración de hogares protegidos a cargo de organizaciones de familiares & Proceso consultivo y deliberativo en la elaboración de los planes nacionales de salud mental \\
\hline
\end{tabular}


Una evaluación realizada por el Ministerio de Salud (MINSAL), como insumo para el tercer plan, muestra que solamente $18 \%$ de las áreas sanitarias realizaba actividades regulares con agrupaciones de usuarios y familiares y otras organizaciones comunitarias $(14,15)$. La percepción de los gestores locales de salud mental es que no se ha logrado una relación sistemática entre los servicios de salud y los usuarios, que permita su participación y el fortalecimiento de sus agrupaciones. Entre las dificultades, se destacan la insuficiente priorización de este tipo de acciones, la escasa horizontalidad en la relación de las instituciones del Estado con las organizaciones sociales y la vulnerabilidad psicosocial de las personas con discapacidad mental y sus familias (14).

El tercer plan nacional de salud mental del 2017 (14) reconoce los avances y dificultades descritos, y propone objetivos y estrategias para reforzar la participación social en salud mental. Por ejemplo, señala el desarrollo de normativas para los equipos de salud en participación comunitaria y fortalecimiento de la asociatividad; la incorporación de usuarios, familiares y otros agentes sociales en los consejos técnicos y consultivos de los servicios de salud mental; y la formación y apoyo a los agentes de salud mental en las comunidades locales. Los planteamientos del plan son sinérgicos con la legislación chilena reciente sobre asociaciones y participación ciudadana (14).

La elaboración del tercer plan (14) y su modelo de gestión (15) fueron liderados por el MINSAL profundizando la participación de profesionales de salud e intersector, usuarios y organizaciones sociales. Este proceso propició intercambios de experiencias, cuestionamientos y deliberaciones sobre cómo desarrollar los servicios de salud mental y mejorar las respuestas a las necesidades de la población.

\section{Políticas y planes nacionales}

Al retorno a la democracia, en 1990, Chile se encontraba con una oferta de salud mental centrada en cuatro instituciones psiquiátricas, que disponían de $74 \%$ del presupuesto. Influenciado por la Declaración de Caracas (16), el MINSAL formula el primer plan nacional de salud mental (11), crea una dirección nacional de salud mental e instala direcciones similares en todas las áreas sanitarias del país para la gestión territorial de servicios. El plan define el desarrollo de la salud mental en la APS y una red de servicios de especialidad en todas las áreas sanitarias. Además, propicia la instalación de centros de salud mental comunitarios y la reinserción social de las personas con discapacidad psíquica internadas en hospitales psiquiátricos (11). Este plan estableció objetivos y opciones estratégicas que persisten hasta hoy.

El segundo plan nacional (12) define con mayor precisión la red de salud mental comunitaria, coloca en el centro del modelo a la APS, asociada al centro de salud mental comunitaria como lugar privilegiado de la especialidad ambulatoria. Promueve también la creación de hospitales de día, la instalación de servicios de psiquiatría en hospitales generales y la desinstitucionalización mediante la creación de programas de rehabilitación psicosocial y hogares protegidos. Con relación a los hospitales psiquiátricos, plantea la transferencia gradual de sus recursos a la red de salud mental. Por primera vez define necesidades presupuestarias y pone como meta la asignación de $5 \%$ del presupuesto de salud para la salud mental, lo que no se logra alcanzar pese al aumento persistente de recursos.

En el año 2005, como parte de un proceso más amplio de reforma del sistema de salud, el MINSAL se dividió en dos subsecretarías, una dedicada a las políticas y regulación, y la otra a la gestión de las redes de atención (17). Este cambio produjo una división de la dirección de salud mental y un debilitamiento de la capacidad de conducción del desarrollo de servicios a nivel nacional y local.

En el año 2017, se lanza el tercer plan nacional de salud mental (14), el cual pone un fuerte foco en la legislación y los derechos humanos, la provisión de servicios, la intersectorialidad, desde la promoción hasta la inclusión social, y la participación, entre otros elementos. Identifica las brechas de servicios $(60 \%$ según estándares del plan previo) y las distorsiones observadas respecto del modelo deseado de red comunitaria. Un ejemplo es la presencia aún hegemónica de tres de los cuatro hospitales psiquiátricos en sus áreas sanitarias; persistencia de personas institucionalizadas; coexistencia de especialidad ambulatoria en hospitales y en la comunidad; insuficiente inclusión de salud mental en el modelo de salud familiar de APS; e insuficiente colaboración intersectorial. Este plan tiene un documento complementario que orienta la gestión local de las redes de servicios de salud mental (15).

La velocidad de implementación de los planes nacionales de salud mental ha variado en el tiempo, por la valoración dada a salud mental por las diversas autoridades del MINSAL y la concurrencia de la reforma a la salud. A nivel de las áreas sanitarias, las diferentes sensibilidades hacia la salud mental de sus direcciones (que gozan de importante autonomía), la actitud favorable o no de los gestores de servicios y hospitales psiquiátricos al proceso de transformación y las diferentes capacidades de gestión de los recursos de salud mental locales, han influido en variaciones geográficas en el desarrollo de los servicios.

\section{Marco legal y regulatorio}

Chile ha realizado avances en la atención de salud mental sin el respaldo de una ley específica en esta materia, aunque sí ha contado con distintas leyes y decretos (18) que incluyen el resguardo de derechos para las personas con enfermedades mentales (cuadro 4).

A pesar de estos avances, en Chile, el nivel de cumplimiento de los estándares de la Convención sobre los Derechos de las Personas con Discapacidad de la Organización de las Naciones Unidas (ONU) aún es bajo. Existen leyes que contravienen dichos estándares (19) y las personas con discapacidad psicosocial o intelectual "están expuestas a situaciones de discriminación y exclusión social, constituyendo un problema social de importancia mayor que el Estado no ha abordado adecuadamente aún" (19). No existe un organismo autónomo que supervise los procedimientos involuntarios; las comisiones de protección existentes carecen de autonomía, atribuciones y recursos suficientes (19); algunos hogares protegidos se han transformado en espacios de discriminación, exclusión, maltrato e institucionalización (20). Además, los equipos de salud mental comunitaria no respetan algunos derechos de los usuarios ni los apoyan suficientemente en sus procesos de inclusión social (21).

La necesidad de una ley de salud mental ha sido demandada desde los profesionales, la sociedad civil y parlamentarios. El intento más avanzado fue entre el 2014 y 2017, con la conformación 
de un grupo de trabajo en el MINSAL que formuló una propuesta de ley acorde a los estándares internacionales. Esta aborda la intersectorialidad (desde la promoción hasta la inclusión social), el derecho a servicios cercanos e inclusivos, la paridad de beneficios entre la salud mental y la salud física, y los mecanismos de monitoreo (22). Si bien se logró elevar el nivel de interés social por el tema y varios candidatos presidenciales lo incluyeron en sus programas, aún no se obtiene el apoyo político necesario para su impulso desde el ejecutivo.

\section{Acceso y calidad de la atención}

Mejorar el acceso y la calidad de la atención en salud mental implicó el desarrollo de regulaciones en la asignación de los recursos financieros, un mayor énfasis en la formación y distribución del recurso humano, y la generación de directrices técnicas específicas.

La principal estrategia financiera para aumentar el acceso a servicios de salud mental ha sido la asignación de las expansiones del presupuesto al desarrollo de los servicios comunitarios. De esta manera, la proporción destinada a los hospitales psiquiátricos disminuyó de $74 \%$ a $21 \%$ en 25 años, y los servicios comunitarios aumentaron a $79 \%$ del presupuesto de salud mental (23).

El mecanismo de financiamiento de salud evolucionó hacia un pago prospectivo asociado a resultado, como una forma de avanzar en los incentivos financieros para mejorar el acceso y la calidad de atención. La salud mental se ha incorporado progresivamente a estos incentivos, tanto en APS como a nivel de especialidad (cuadro 5).

Por otra parte, la ley de garantías en salud (2005) regula los seguros público y privado al establecer las mismas condiciones para acceso, oportunidad, calidad y protección financiera de 80 problemas de salud, entre ellos esquizofrenia, depresión, trastorno bipolar y consumo perjudicial de alcohol y drogas (24).

Los mecanismos de financiamiento que se han implementado constituyen un avance al permitir visibilizar y controlar la actividad, con resguardo del uso efectivo del presupuesto asignado para salud mental, aunque persista siendo insuficiente a la luz de la demanda de atención.
No resulta tan claro si dichos mecanismos de pago han impactado en forma positiva como un incentivo a la articulación entre el nivel primario y los niveles secundario/terciario y la continuidad de cuidados.

Al mismo tiempo, las competencias en gestión por parte de algunos equipos de salud mental son aún insuficientes, lo que menoscaba la administración de recursos y produce desviaciones en la aplicación del modelo comunitario. Se han formulado reglamentaciones para mitigar esas desviaciones y asegurar el acceso, equidad y calidad (15).

La dirección nacional de salud mental ha generado documentos técnicos, con la cooperación de expertos, para promover estándares de calidad en la organización de la red de servicios, así como en las prestaciones entregadas a los usuarios. Destacan las orientaciones técnicas para las consultorías de salud mental, actividades que reúnen a profesionales de especialidad y APS para la resolución de casos complejos; los consejos técnicos, en los cuales los actores claves de las redes territoriales de salud mental toman decisiones para responder a las necesidades de la población; y los comités de gestión

\section{CUADRO 4. Principales componentes del marco legal y regulatorio para personas con trastornos o discapacidades mentales en Chile}

\begin{tabular}{|c|c|}
\hline Leyes & Decretos del Ministerio de Salud \\
\hline $\begin{array}{l}\text { Garantías en salud (2004): permite acceso, oportunidad, calidad y protección } \\
\text { financiera en } 80 \text { problemas de salud (cuatro de ellos relacionados con la salud mental) }\end{array}$ & $\begin{array}{l}\text { Funcionamiento de comisiones nacional y regionales de protección de personas con } \\
\text { enfermedad mental (2000 y 2012): velan por los derechos la atención de salud }\end{array}$ \\
\hline $\begin{array}{l}\text { Convención sobre los Derechos de las Personas con Discapacidad (ONU), } \\
\text { ratificada por Chile (2008) }\end{array}$ & $\begin{array}{l}\text { Regulación de la hospitalización de las personas con enfermedades mentales y los } \\
\text { establecimientos que la proporcionan (2000) }\end{array}$ \\
\hline Inclusión social de personas con discapacidad (2010) & Normas para el funcionamiento de nuevas estructuras comunitarias, tales como: \\
\hline $\begin{array}{l}\text { Derechos y deberes en la atención en salud (2012): regula tratamientos invasivos, } \\
\text { hospitalizaciones involuntarias, uso de aislamiento o contención, consentimiento, } \\
\text { investigaciones y comisiones de protección }\end{array}$ & $\begin{array}{l}\text { - hogares protegidos }(2000) \\
\text { - residencias protegidas }(2006) \\
\text { - centros de salud mental comunitaria (2008). }\end{array}$ \\
\hline \multicolumn{2}{|l|}{$\begin{array}{l}\text { Inclusión laboral (2017): obliga a empresas con } 100 \text { o más trabajadores a contratar } \\
\text { al menos 1\% de personas con discapacidad }\end{array}$} \\
\hline \multicolumn{2}{|c|}{$\begin{array}{l}\text { ONU, Organización de las Naciones Unidas. } \\
\text { Elaboración propia } 2018 \text { en base a documentos disponibles en la Biblioteca del Congreso Nacional de Chile, } 2018 .\end{array}$} \\
\hline \multicolumn{2}{|c|}{$\begin{array}{l}\text { CUADRO } 5 \text {. Mecanismos financieros utilizados en la actualidad en el sistema público de salud que regulan los servicios de } \\
\text { salud mental }\end{array}$} \\
\hline Atención primaria de salud & Nivel de especialidad \\
\hline \multirow{2}{*}{$\begin{array}{l}\text { Pago capitado (activo desde 2001): asignación de fondos por número de } \\
\text { personas inscritas en cada centro de APS. La canasta de prestaciones incluía } \\
\text { consulta de salud mental e intervención psicosocial grupal. } \\
\text { Plena integración de salud mental en el financiamiento per cápita (activo desde } \\
\text { 2015): Iogra la paridad con la salud física en mecanismos de financiación. } \\
\text { Integra todas las acciones del programa de salud mental integral de APS. }\end{array}$} & $\begin{array}{l}\text { Prestaciones valoradas (activo desde 2002): convenio entre el seguro público y las áreas } \\
\text { sanitarias que establece un número anual de paquetes o canastas de prestaciones. } \\
\text { El paquete incluye actividades profesionales en establecimientos y en la comunidad, } \\
\text { medicamentos, exámenes, hospitalización, etc. }\end{array}$ \\
\hline & $\begin{array}{l}\text { Salud Mental cuenta actualmente con } 42 \text { paquetes asociados con: } \\
\text { - diagnóstico (ej. todas las prestaciones para depresión grave en un año), } \\
\text { - tratamiento (ej. tratamiento ambulatorio de adicciones en menores de } 18 \text { años), } \\
\text { - atención cerrada (ej. día cama hospital corta estadía, día hogar protegido, etc.), } \\
\text { - peritajes judiciales. }\end{array}$ \\
\hline
\end{tabular}


de camas, orientados a optimizar el uso de estos recursos (15). En el caso de los cuatro problemas de salud mental contemplados en la ley de garantías en salud, cada uno tiene una guía clínica con las prestaciones psicosociales y biomédicas (24).

El reciente desarrollo de los modelos de gestión de la red de salud mental (15) y de los distintos establecimientos (25, 26), que incluyen principios y criterios para su instalación y funcionamiento, se espera que sean una contribución para una mayor coherencia de la inversión pública con el modelo comunitario.

Con relación a los recursos humanos, ha sido relevante la incorporación de psicólogos en la APS, los que actualmente alcanzan a 12,8 por 100000 habitantes. La capacitación de los equipos de salud general, profundizada con la aplicación del programa de la OMS para superar brechas en salud mental (mhGAP, por sus siglas en inglés), ha alcanzado a más de 3000 profesionales, técnicos y gestores comunitarios entre 2016 y 2017 (27). La ampliación del financiamiento para formar especialistas por parte del MINSAL ha aumentado la disponibilidad de psiquiatras y creado nuevos programas de formación acordes con el modelo comunitario (14).

En capacitación, ha sido importante la alianza entre el MINSAL y la Escuela de Salud Pública de la Universidad de Chile, que desde hace 16 años imparte diplomas de postgrado en competencias clínicas y de gestión en salud mental comunitaria, con más de 1600 egresados a la fecha (28).

\section{Sistema nacional de información}

El sistema de información se ha construido de manera progresiva junto con la implementación de los planes nacionales $\mathrm{y}$ en forma integrada con el sistema general de información en salud. Antes de 1993, solo se disponía de datos sobre psiquiatría, número de consultas ambulatorias y ocupación de camas en hospitales generales y psiquiátricos. Desde ese año se registra el número de consultas individuales y sesiones grupales de salud mental en APS asociado con datos demográficos. Esta información ha sido útil para monitorizar el proceso de integración de la salud mental en APS. Posteriormente, se diversificó la información disponible a distintos tipos de prestaciones, profesionales y establecimientos.
Un hito crucial en el desarrollo del sistema de información fue la incorporación en el 2004 del registro de ingresos y egresos, y del número de consultantes en tratamiento activo, diferenciados por el diagnóstico principal (29). Esto ha permitido conocer las tasas de acceso, las brechas de tratamiento y las inequidades geográficas.

En la actualidad, este sistema está en proceso de consolidación, apoyado por tecnologías de información, sobre todo el registro electrónico que se está instalando en forma gradual en muchos lugares del país. Esto ha contribuido a disminuir los errores y la falta de información, los cuales eran uno de los principales problemas en años anteriores.

\section{FACILITADORES Y OBSTACULIZADORES DE LA GOBERNANZA}

Disponer de una visión estratégica de cómo desarrollar los servicios para implementar el modelo comunitario de salud mental ha sido uno de los factores críticos para los logros alcanzados en Chile. Tal como ha sido destacado para algunos países de África y Asia $(5,9,10)$, la gobernanza debería incluir políticas nacionales con una clara visión estratégica. En Chile, esto se ha favorecido por la mantención de un mismo cuerpo central de ideas a lo largo de tres décadas, lo que permitió, además, su enriquecimiento conceptual y operacional desde las experiencias locales. Un aspecto central de la visión, desde la Declaración de Caracas y en los tres planes, ha sido la integración de la APS como el principal proveedor de salud mental.

La puesta en práctica de la visión estratégica se ha facilitado con la instalación y permanente refuerzo de direcciones de salud mental en cada área sanitaria. Estas han desarrollado competencias crecientes para gestionar los recursos, impulsar el desarrollo de servicios, coordinar las redes de atención y generar espacios de diálogo y toma de decisiones con los actores locales, siempre con una orientación hacia la mejora de respuestas hacia la población. De manera similar a lo que se describe en la literatura (8), la comunicación a nivel local entre distintos equipos de salud y representantes de usuarios y otros sectores ha contribuido a reforzar una visión común y un clima de colaboración.
Las estrategias de gobernanza no hubieran tenido el mismo impacto si no se hubiera dispuesto de un presupuesto creciente para salud mental, asignado en su mayor parte a los nuevos servicios comunitarios y con definición de costos para las distintas prestaciones, concordante con lo que ha sugerido Chisholm (30) para la implementación local de planes y el aumento de servicios en países de bajo y mediano ingreso.

La integración incremental de la salud mental al sistema público de salud, tanto a nivel de políticas, regulaciones y gestión nacional, como a nivel local en APS, hospitales generales y centros ambulatorios de especialidad, ha sido fundamental para dar sustentabilidad a largo plazo a los servicios y disminuir la estigmatización de la enfermedad mental. Estos aspectos han sido también planteados por otros autores que han abordado aspectos de gobernanza en salud mental $(10,31)$.

Siddiqi (9) ha destacado el rol del sistema de información en la gobernanza de los servicios de salud, al cual considera esencial para la comprensión del funcionamiento y la toma de decisiones. Esto es válido para el sistema de salud mental en Chile, donde las direcciones de salud mental nacional y locales disponen de información sobre aspectos básicos de acceso, calidad de atención y equidad.

Entre las debilidades que aún tiene la gobernanza en salud mental en Chile, destacan en especial dos dimensiones: participación y legislación. Los obstáculos para avanzar en ellas son de naturaleza política. La escasa participación de los usuarios y organizaciones comunitarias puede explicarse, en parte, por el bajo nivel de asociatividad y participación que existe en general en el país, sumado al efecto de la reforma de salud que puso mayor énfasis en aspectos técnicos que en los aportes de la ciudadanía (32). El escaso empoderamiento efectivo para la toma de decisiones, el aislamiento social y la baja participación de los usuarios son contradictorios con lo que plantea Díaz-Castro para una adecuada gobernanza de los programas de salud mental (8). La legislación es uno de los pilares de la gobernanza en salud (9). Chile, a diferencia de otros países latinoamericanos (33-35), ha realizado una de las experiencias exitosas de implementación del modelo comunitario de salud mental sin disponer de una ley específica. El principal obstáculo ha sido no contar con respaldo suficiente de los poderes ejecutivo 
y legislativo. Esta falencia parece haber sido compensada, al menos durante estas tres décadas, por una serie de circunstancias particulares del país, tales como la continuidad en el gobierno de la misma coalición política por 20 años, el desarrollo económico y la adherencia de la mayoría de las áreas sanitarias a las políticas centrales. El desafío mayor es disponer de una ley que garantice a futuro los derechos de las personas con problemas de salud mental y que asegure financiamiento y concordancia con el modelo comunitario.

\section{CONCLUSIONES}

La experiencia chilena confirma la importancia de políticas nacionales con visión estratégica, articuladas con legislación, participación y sistema de información, para la gobernanza en salud mental. Similares observaciones se han realizado en algunos países de África y Asia $(5,9,10)$. Los mecanismos de gobernanza podrán variar en los distintos países según los contextos políticos, la forma de funcionamiento de los sistemas de salud y las ventanas de oportunidades de los diferentes momentos históricos.

Esta experiencia, en pos de su consolidación, enfrenta la necesidad de profundizar el proceso, especialmente en las áreas de participación y legislación, así como de perfeccionar y utilizar el sistema de información para mejorar la equidad y la calidad del trabajo en salud mental. Estas estrategias contribuirían a reducir el impacto negativo de gestores locales con escasas competencias o resistentes a priorizar el tema.

Conflicto de intereses. Mauricio Gómez, Rodrigo Díaz, Viviana Hernández y Susana Chacón presentan filiación institucional con el Ministerio de Salud de Chile. Matías Irarrázabal tiene filiación institucional con la Oficina Panamericana de la Salud/Organización Mundial de la Salud.

Declaración. Las opiniones expresadas en este manuscrito son responsabilidad del autor y no reflejan necesariamente los criterios ni la política de la RPSP/ PAJPH y/o de la OPS.

\section{REFERENCIAS}

1. World Health Organization. Everybody's business--strengthening health systems to improve health outcomes: WHO's framework for action. Geneva: World Health Organization, 2007. Disponible en: http://www.who.int/iris/handle/ 10665/43918

2. Pan American Health Organization (PAHO). 154th Session of the Executive Committee, Strategy for Universal Health Coverage. Resolution CE154/12. Washington, DC: PAHO; 2014. Disponible en: https://tinyurl.com/y75tm4xw Acceso el 24 de mayo de 2018.

3. Pan American Health Organization (PAHO). Plan of Action on Mental Health 2015-2020 [Internet]. 53rd Directing Council of PAHO, 66th Session of the Regional Committee of WHO for the Americas. Washington, D.C.: PAHO; 2014 (Documento CD53/8, Rev. 1 and Resolution CD53.R7). Disponible en: http: / / www2.paho.org / hq / index. php?option $=$ com_docman \& task $=$ doc download\&gid=26722\&Itemid=270\&lang=en Acceso el 24 de mayo de 2018.

4. World Health Organization. Comprehensive Mental Health Action Plan 2013-2020. Geneva: World Health Organization, 2013. Disponible en: http://apps.who.int/gb/ ebwha/pdf_files/WHA66/A66_R8-en.pdf

5. Petersen I, Marais D, Abdulmalik J, Ahuja S, Alem A, Chisholm D, et al. Strengthening mental health system governance in six low- and middleincome countries in Africa and South Asia: challenges, needs and potential strategies. Health Policy Plan. 2017;32(5):699-709.

6. Hailemariam M, Fekadu A, Selamu M, Medhin G, Prince M, Hanlon C. Equitable access to integrated primary mental healthcare for people with severe mental disorders in Ethiopia: a formative study. International Journal for Equity in Health. 2016;15:121.
7. Araya R, Zitko P, Markkula N. The impact of universal health care programmes on improving 'realized access' to care for depression in Chile. Administration and Policy in Mental Health and Mental Health Services Research. 2018. Disponible en: https: / /link.springer.com/ article/10.1007/s10488-018-0864-z\#citeas

8. Díaz-Castro L, Arredondo A, PelcastreVillafuerte BE, Hufty M. Governance and mental health: contributions for public policy approach. Revista de Saude Publica. 2017:51:4.

9. Siddiqi S, Masud TI, Nishtar S, Peters DH, Sabri B, Bile KM, et al. Framework for assessing governance of the health system in developing countries: gateway to good governance. Health Policy (Amsterdam, Netherlands). 2009;90(1):13-25.

10. Marais DL, Petersen I. Health system governance to support integrated mental health care in South Africa: challenges and opportunities. Int J Ment Health Syst. 2015;9:14.

11. Ministerio de Salud de Chile. Políticas y Plan Nacional de Salud Mental. Santiago, Chile. 1993.

12. Ministerio de Salud de Chile. Plan Nacional de Salud Mental y Psiquiatría. Santiago, Chile; 2000.

13. Minoletti A, Alvarado R, Rayo X, Minoletti M. Sistema de salud mental de Chile: segundo informe (Organización Mundial de la Salud-Informe sobre la evaluación de los sistemas de salud mental). Ministerio de Salud de Chile; 2014.

14. Ministerio de Salud de Chile. Plan Nacional de Salud Mental 2017-2025. Santiago, Chile. 2017.

15. Ministerio de Salud de Chile. Modelo de gestión: red temática de salud mental en la red general de salud. Santiago, Chile; 2018.

16. Levav I, Restrepo H, de Macedo CG. The restructuring of psychiatric care in Latin America: a new policy for mental health services. Journal of Public Health Policy. 1994;15(1):71-85.

17. Decreto con Fuerza de Ley: fija texto refundido, coordinado y sistematizado del Decreto Ley $\mathrm{N}^{\circ} 2$ 763, de 1979 y de las Leyes No 18933 y No 18 469, 2006, Chile.

18. Díaz E. Estudio comparado de leyes de salud mental. Ministerio de Salud Santiago, Chile; 2015.

19. Observatorio de Derechos Humanos de las Personas con Discapacidad Mental. Derechos humanos de las personas con discapacidad mental: diagnóstico de la situación en Chile. Santiago, Chile; 2014.

20. Chuaqui RJ, Salas A, Ríos U, Salas R, Ávila A. Informe del FONIS SA11I2120: Cumplimiento de objetivos y acciones de rehabilitación según norma técnica $\mathrm{N}^{\circ} 53$ para personas con discapacidad psíquica en hogares protegidos de la región de Valparaíso, mediante significaciones de usuarios y administrativos, y propuestas de mejoramiento. Valparaíso, Chile; 2014.

21. Minoletti A, Toro O, Alvarado R, Carniglia C, Guajardo A, Rayo X. Respeto a los derechos de las personas que se atienden en servicios de psiquiatría ambulatoria en Chile. Rev Med Chile. 2015;143:1585-92.

22. Grupo de Trabajo Proyecto de Ley de Salud Mental. Ley de Salud Mental para Chile: informe del grupo de trabajo. Santiago, Chile: Ministerio de Salud; 2016.

23. Minoletti A. The reform of mental health services in Chile: 1991-2015. L'information psychiatrique. 2016;92(9):761-6.

24. Decreto $N^{\circ} 3$ : Aprueba Garantías Explícitas del Régimen General de Garantías en Salud, 2016, Chile.

25. Ministerio de Salud de Chile. Modelo de gestión: unidad de hospitalización de cuidados intensivos en psiquiatría para población adulta e infantoadolescente (UHCIP). Santiago, Chile; 2016.

26. Ministerio de Salud de Chile. Modelo de gestión: centro de salud mental comunitaria. Santiago, Chile; 2018. 
27. Ministerio deSalud deChile. Orientaciones técnico-administrativas de salud mental para la atención primaria. Santiago, Chile; 2018.

28. Universidad de Chile, Escuela de Posgrado, Facultad de Medicina. Proyecto de Magíster en Salud Mental y Psiquiatría Comunitaria. Santiago, Chile; 2017.

29. Ministerio de Salud de Chile. Departamento de Estadísticas e Información de Salud. Santiago, Chile; 2018.

30. Chisholm D, Burman-Roy S, Fekadu A, Kathree T, Kizza D, Luitel NP, et al. Estimating the cost of implementing district mental healthcare plans in five lowand middle-income countries: the PRIME study. Brit J Psychiat. 2016;208(S56):s71-s8.
31. Acuña C, Sepúlveda R, Salgado O. Stigma and psychiatric care in Latin America: its inclusion on the universal health coverage agenda. Brit J Psych International. 2015;12(4):81-3.

32. Montenegro CR, Cornish F. Historicising involvement: the visibility of user groups in the modernisation of the Chilean Mental Health System. Crit Public Health. 2017:1-13.

33. Cohen H, Natella G. Trabajar en salud mental, la desmanicomialización en Río Negro. Buenos Aires: Lugar Editorial; 1995.

34. Gonçalves RW, Vieira FS, Delgado PGG. Política de saúde mental no Brasil: evolução do gasto federal entre 2001 e 2009. Revista de Saude Publica. 2012;46:51-8.
35. Toyama M, Castillo H, Galea JT, Brandt LR, Mendoza M, Herrera V, et al. Peruvian mental health reform: a framework for scaling-up mental health services. IJHPM. 2017;6(9):501-8

Manuscrito recibido el 12 de abril de 2018. Aceptado para su publicación, tras revisión, el 12 de junio de 2018
ABSTRACT

\section{Analysis of Governance in the Implementation of the Chilean Community Mental Health Model}

Keywords Governance; community mental health services; health policy; Chile.
This article describes and analyzes the role of governance in the development of the Chilean community mental health model. It describes and analyzes five dimensions of governance: 1 ) the implementation of a series of strategies to encourage the participation of users of mental health services has yielded limited results; 2) the implementation of three national mental health plans with the same strategic vision over the course of three decades has helped increase access to community care for people with mental illness and diminish the role of psychiatric hospitals; 3) the lack of a mental health law remains a challenge, although a series of laws and decrees has partially compensated for this; 4) financial resource allocation regulations, human resources education, and technical guidelines have helped improve access and the quality of care; and 5) the gradual development of a mental health information system has provided knowledge, primarily on access rates, treatment gaps, and geographical inequities. The Chilean experience confirms the importance of implementing a set of governance strategies and mechanisms to promote the development and operation of mental health services. The type of governance strategies and mechanisms can vary from country to country, depending on the context and windows of opportunity.
RESUMO

\section{Análise da governança na implementação do modelo comunitário de saúde mental no Chile}

\footnotetext{
Palavras-chave
}

O presente artigo descreve e analisa o papel da governança no desenvolvimento do modelo de saúde mental comunitária no Chile. São descritas e analisadas cinco dimensões da governança: 1) o desenvolvimento de uma série de estratégias para favorecer a participação dos usuários de serviços de saúde mental não tem obtido resultados satisfatórios; 2 ) a implementação de três planos nacionais de saúde mental, com a mesma visão estratégica ao longo de três décadas, tem contribuído para melhorar a acessibilidade à atenção comunitária de indivíduos com doenças mentais e restringir o papel dos hospitais psiquiátricos; 3) a inexistência de uma legislação sobre saúde mental continua sendo um desafio, embora tenha sido em parte compensada por distintas leis e decretos; 4) as regulamentações para designação dos recursos financeiros, a capacitação de recursos humanos e as diretrizes técnicas têm servido para melhorar o acesso e a qualidade da atenção e 5) a construção progressiva de um sistema de informação em saúde mental tem possibilitado conhecer sobretudo o nível de acesso, as lacunas de tratamento e as desigualdades geográficas. A experiência chilena confirma a importância do uso de um conjunto de estratégias e mecanismos de governança para favorecer o desenvolvimento e o funcionamento dos serviços de saúde mental. Várias estratégias e os mecanismos de governança podem ser usados em cada país de acordo com o contexto e a janela de oportunidades.

Governança; serviços comunitários de saúde mental; política de saúde; Chile 